\title{
Enlarging the scope: grasping brain complexity
}

\author{
Emmanuelle Tognoli ${ }^{*}$ and J. A. Scott Kelso ${ }^{1,2}$ \\ 1 The Human Brain and Behavior Laboratory, Center for Complex Systems and Brain Sciences, Florida Atlantic University, Boca Raton, FL, USA \\ ${ }^{2}$ Intelligent System Research Centre, University of Ulster, Derry, Northern Ireland
}

Edited by:

Emili Balaguer-Ballester,

Bournemouth University and

Bernstein Center for Computational

Neuroscience

Heidelberg-Mannheim, UK

Reviewed by:

Nicholas M. Dotson, Montana State University, USA

Karl Friston, University College

London, UK

\section{*Correspondence:}

Emmanuelle Tognoli, The Human

Brain and Behavior Laboratory,

Center for Complex Systems and

Brain Sciences, Florida Atlantic

University, 777 Glades Road, Boca

Raton, FL-33431, USA

e-mail: tognoli@ccs.fau.edu
To further advance our understanding of the brain, new concepts and theories are needed. In particular, the ability of the brain to create information flows must be reconciled with its propensity for synchronization and mass action. The theoretical and empirical framework of Coordination Dynamics, a key aspect of which is metastability, are presented as a starting point to study the interplay of integrative and segregative tendencies that are expressed in space and time during the normal course of brain and behavioral function. Some recent shifts in perspective are emphasized, that may ultimately lead to a better understanding of brain complexity.

Keywords: metastability, brain coordination dynamics, integration, segregation, functional connectivity

\section{THEORIES OF THE BRAIN: A CONCISE HISTORY}

How does the brain work? This nagging question is an habitué from the top ten lists of enduring problems in Science's grand challenges. Grasp this paradox: how is one human brain-a chef d'oeuvre of complexity honed by Nature-ever to reach such a feast as to understand itself? Where one brain may fail at this notorious philosophical riddle, may be a strong and diversely-skilled army of brains may come closer. Understanding of the local principles at play has emerged due to the combined efforts of many scientists: neurons talk to their partners by teasing them with charged particles of either excitatory or inhibitory effect, as Nobel laureate Sir John Eccles demonstrated (Eccles, 1966). Targeted release of ions was later shown at sites that seem designed for the exchange of information: typically the axonal termination of the emitting neuron facing the dendrites of a receiving partner (Hodgkin and Huxley, 1952). Many of those two-some neural interlocutors build into a reticulum with remarkable emergent properties. A booming network science followed, generalizing microscale principles on a large-scale. David Rumelhart and James McClelland, Stephen Grossberg and many others pursued this connectionist endeavor (Grossberg, 1976; Rumelhart and McClelland, 1986). Putting function first, they aimed to model specific aspects of human cognition and behavior such as visual perception or language. Yet others, such as Olaf Sporns have devoted much effort to the neurobiological fidelity of their inquiries, conceiving behavior as an emergent phenomenon from an appropriate connectional design (Sporns et al., 2004). The latter may be probed both with theoretical connectivity models where brain complexity is carefully thought through (Sporns et al., 2000) along with empirically-derived models that borrow their connectional blueprints from images of "real" brains (Honey et al., 2010). Though brief, this historical overture suggests that the brain has demonstrable mechanisms for both point-to-point communication of information - most obviously at the microscale—as well as emergent phenomena arising from network properties at the meso- and macroscale.

\section{NEURONAL RELAYS AND THE PROPAGATION OF INFORMATION}

The principle of synaptic transmission proved to be pictureperfect for a theory of communication, boosted by the influential work published in 1948 by Claude Shannon (Shannon, 1948). Transfer of information became a principal tenet of brain function, and theories went so far as to conceive of "centers" (Charcot, 1878; see perspectives in Catani and ffytche, 2005) as final destinations for information to be communicated (the concept has now retreated, although it remains perniciously present in neuroscientists' conceptions of brain hierarchies ${ }^{1}$ (e.g., Meunier et al., 2010); an alternate view is that it is the journey,

\footnotetext{
${ }^{1}$ Clearly, the brain is not a diffuse web of randomly coupled dynamical systems. It is a highly structured organ that has been crafted by evolution. The outcome of phylogenesis, a slow timescale pattern-forming process (Bressler and Tognoli, 2006) is the progressive emergence of functional specialization, where some brain regions take on a more integrated role than others, a phenomenon that has been named hierarchical organization (see Robert, 1999; Zilles et al., 2002 for anatomo-functional patterning, see also Honey et al., 2007 for a related network perspective). The notion of hierarchy is popular in neuroscience and may be relevant (see also Kelso and Tuller, 1981 for a discussion of heterarchical and coalitional forms of brain organization): hierarchical systems induce a distinction between ascending and descending connections that could contribute to symmetry breaking which may then lead to directed coupling and information transfer (Tognoli and Kelso, 2014).
} 
but not an elusive final destination, that really matters). This theory of information processing in the brain raises a question that may not have received enough attention: can we readily transpose findings from the smallest synaptic level-findings that speak of only a pair of neurons - to larger spatial scales such as neural areas or the whole brain? In all justice, countless emergent phenomena were discovered through this extrapolation, both in empirical and theoretical work (e.g., Sperry, 1969; Hopfield, 1982; McLeod et al., 1998). But it remains an uneasy feeling that so much of Brain Science is built upon the foundation of a pair of neurons, outside the context of their networks, and with two open-ended areas of darkness at either of their extremities that must be thought of as the entire remainder of the organism's brain (and body). ${ }^{2}$

\section{COLLECTIVE POWER OF NEURONAL SYNCHRONY}

We will come back to information transmission later, but let us now explore the matter of spatial scales. As humans tend to agree, increased size makes up for smarter brains (disclosure: both authors are human), and those bigger brains have room to organize themselves at multiple levels, coalescing into functional ensembles at several steps along the way up from neurons to functional areas and to the entire brain (Kelso, 1995; Bressler and Tognoli, 2006; Buzsáki, 2007; Kelso et al., 2013; Tognoli and Kelso, 2013). At larger and more integrated levels of description, other ordering phenomena were discovered that brain scientists conceive in terms of information exchange. In the late 1980's, two groups of scientists, one with Reinhard Eckhorn (Eckhorn et al., 1988) and another with Charles Gray and Wolf Singer (Gray and Singer, 1989), discovered that perceptual integration

\footnotetext{
${ }^{2}$ Although much of neuroscience and its methodology focus on communication between pairs of neurons at the synaptic level, there is a long tradition of trying to understand the brain in terms of self-organization and dynamic coordination. For instance, Ross Ashby theorized on regulatory brain systems, considered under an equilibrium (homeostatic) perspective (Ashby, 1962) and he contributed to developing Cybernetics ("steermanship" born from control theory). From a more physical perspective, Arthur Iberall's Homeokinetics (formulated as Homeodynamics for biology) stressed the persistent, marginally stable nature of open thermodynamic systems which necessarily organize themselves as cyclic, physical action modes at all scales (see Yates, 2008, for review; also Eigen and Schuster, 1979 for a more formal but related approach). More recent years have seen the emergence of Synergetics as a foundational mathematical framework for nonequilibrium self-organization in physics, chemistry and biology (Haken, 1983). Synergetic self-organization is one of the cornerstones of Coordination Dynamics which stresses adaptive, informationally meaningful, bidirectional couplings on multiple levels (Kelso, 1995; Kelso and Haken, 1995; Kostrubiec et al., 2012; Kelso et al., 2013). A key notion of Synergetics that overcomes simple directional interactions between pairs of neurons is so-called circular or reciprocal causality (see discussions in Kelso, 1995; Kelso and Engstrøm, 2006; Tognoli and Kelso, 2013). In a multiscale perspective, circular causality manifests itself in terms of the micro and macroscopic states of a system reciprocally affecting each other; such that the macroscopic (ensemble) behavior enslaves the microscopic states, the ensemble average of which in turn produces the macroscopic states. It is possible that the formalism called for in this essay will rest upon outstanding developments along these lines. On the other hand, since "macro" and "micro" are relative terms in neurobiological dynamical systems where the timescales are not well-separated, a more systematic level by level approach may be required (see Kelso et al., 2013).
}

(or Gestalt) elicited transiently synchronous action potentials amongst neurons that had shared-stakes in the sensory object being viewed. Those neurons dealt with separate parts of the visual field, and they generally disagreed on when to elicit their action potentials in the regular course of their participation in visual function. Somehow however, through the complex labyrinth of the visual cortex and despite the fact that some finite amount of time was required to get from any one to any other of them (delays and frustrations manifested in their usual asynchrony), they managed to coincide when they responded to the same object. What we knew from those neurons is that they "responded" strongly to orientation, fragments of contours with sharp luminance gradients. Their synchrony it seems, was a trace of their joint participation in the construction of something bigger (the object) than what each of them was about (pieces of contour). These discoveries resonated with earlier theorizing regarding the organized behavior of neurons such as Donald Hebb's cell assemblies (Hebb, 1949) or Walter Freeman's mass action (Freeman, 1975). The findings by Eckhorn, Singer and Gray launched a relentless quest for synchrony in all parts of the brain and for numerous functions (von der Malsburg et al., 2010), and took the form of several variants (the most basic being coincidence of action potentials and phase-locking of neural oscillations).

\section{IRRECONCILABLES}

Theories and dedicated experimental paradigms were built upon both discoveries of synaptic transmission and neural synchronization. And from each side, supporting evidence abounded (see previously mentioned references for evidence and reviews). In spite of their prominence and ubiquity though, the theories carefully avoided confrontation with each other, remaining mostly in the separate territories of distinct research groups. ${ }^{3}$ One may note already some difficulties in reconciling them. Let us follow the two extreme views: perfect synchronization and perfect transfer. If all neurons were completely synchronized, they would remain in a changeless state of simultaneity. It is unclear how this system could have flows of information from one place to another. On the other end, if each neuron relayed information in a strict sense, the system would lack basic simultaneity through which synchronous phenomena could emerge. In their radical form it seems, the theories of information exchange qua synaptic transfer or neural synchrony are mutually exclusive.

\section{CAN WE FIND DIRECTIONS IN THE BRAIN?}

The tension is also visible in some empirical facts. Although directed flows of information in the Shannonian spirit do most certainly occur in neural networks, it is indeed quite challenging to track information otherwise than in local or statistical sense (by tracking, we mean to follow the path of information on a brain map as one would follow any object in motion on a symbolic representation of its spatial domain-see Figure 1). The

\footnotetext{
${ }^{3}$ There are few exceptions, for instance Fries' (2005) theory of communication through coherence that uses the phase of macroscopic patterns of coherence as a scaffold for selective modulation of information transfer at the microscopic level.
} 


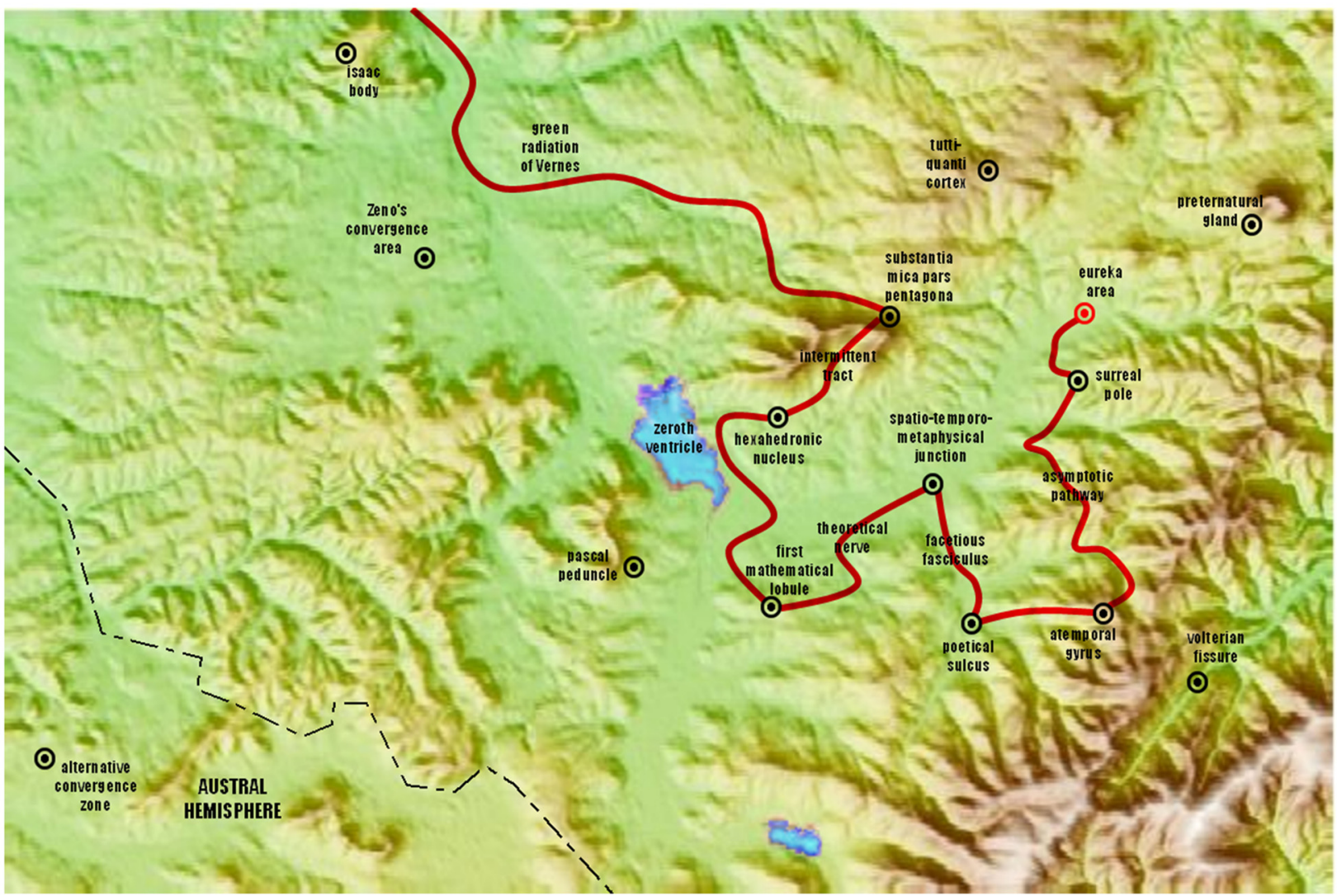

FIGURE 1 | A teasing figure aimed at marking difference between directionality in well-formed Shannonian systems (as in this imaginary brain map) and in complex systems such as the brain. In the latter, direction is less intuitive past immediate spatial and temporal neighborhoods, and it can reverse across spatial scales of observation (Tognoli and Kelso, 2013). The question is highly relevant though, when one is concerned with where and how to effect changes in the system. brain network after all, is a web, as Francisco Varela et al. (2001) emphasized, and one gets quickly lost with all the branchings, loops and loops within loops (Edelman, 1993; Gallos et al., 2012); structural features that "distribute" information (albeit unlike a postmaster distributes mail). So it seems that transmission principles do not scale well upward from simple "channels" of synaptic interactions to the larger and more complex web of evolved brains. Thus, it is without surprise that the brain betrays an essential communicational etiquette: its parts do not behave in a sequential-one-talks-at-a-time-manner (as opposed to the humoristic illustration of Figure 1). It is also overwhelmingly clear that "inputs" from the environment do not enter a silent system. Brain parts constantly exchange information about their current and past affairs, and what comes in at a given time works more as a "perturbation" to an already established ballet, an event that weaves itself within a broader scheme of coordinated brain behavior rather than the sole commander of all things present (Kelso, 1995). All of these nuances differentiate the brain from a channel in which information is transferred from sender to receiver. This situation creates mounting complications. The quest for directional flows in the brain has proved difficult both conceptually and methodologically, yet, it has not deterred efforts toward understanding. Mathematical and empirical studies aimed at resolving these questions are an active area pursued by many, including our own colleagues (Bressler et al., 2007; Bressler and Seth, 2011).

\section{BRAIN ORGANIZATION: SYNCHRONIZATION OR COORDINATION?}

The second concept, synchrony, also bears its share of ambiguities. The firm ground on which we stand is that the timing of neural activity is not left to hazard (as if parts of the brain behaved independently, and were totally oblivious of what the others were doing). "When" one brain part behaves influences when others do. And like social creatures, neurons also use the power of their numbers to increase their impact, creating collective structures that speak from a common voice. A generic name for such behavior is coordination (Kelso, 1995). Synchrony is a narrower concept, one of several ways for a system to coordinate itself. Though synchrony has multiple meanings (and though its study uses a variety of tools across the board), it is easy to conceive and to model, perhaps explaining its systematic resort. To be rigorous however, synchrony requires two important and inter-related characteristics: first, that the underlying temporal 
order in which the system is embedded be frequency-locked, and second, that attractors have emerged in the system's coordination dynamics (attractors are mathematical structures that entrap the system's coordination dynamics into persistent-hard to breakstates). How to examine if there are attractors in the system from empirical data? We do know how to go from theoretical descriptions of dynamics with- and without-attractors to their phenomenology (using models to create data at will), but we are not very successful at taking the return path: demonstrating attractors or lack thereof from looking at data, at least for complicated systems like the brain. The other criterion, frequencylocking, is a little bit easier. And what the data say is that brain parts exhibit tendencies toward frequency-locking without going all the way to being perfectly identical. So it seems, we could be dealing with synchronous tendencies rather than synchrony (Kelso, 2008; Tognoli and Kelso, 2014). The difference may seem subtle to some (and some might be tempted to brush it aside as noise or measurement uncertainty), but mathematically and conceptually, it is enormous: it speaks of two entirely different species of dynamical systems, as said before, one with and the other without attractors. We are turning the spotlight to this distinction because we believe it to be paramount for progress in understanding the brain.

The mathematical formalism of "generalized synchronization" (Rulkov et al., 1995; see also Barreto et al., 2000) is apt to quantify such regimes of coordination sans attractors. Generalized synchronization assumes the existence of a functional mapping between two dynamical processes, without imposing the strong constraint of identical synchronization usually sought for in classical measures of coherence and correlation. The notion of generalized synchrony is set to grow in importance in the context of a coordination dynamic between weakly coupled components-a beneficial situation that enhances system complexity. The absence of complexity is a nefarious situation that André Gide related to tyranny.

\section{AT THE CROSSROADS OF PROPAGATION AND SYNCHRONIZATION}

We hope that the previous exposé motivated the thought that neural networks neither operate on perfect synchrony nor on strict transfer, which is good news as each prevents expression of important features of the other. What then is the link between them? Some attempts at studying synchrony and transfer in a common formalism have emerged, exceptions to their usual avoidance of one another. One is the quest for quantifying directional coupling as discussed above. Another attempt is functional (relatedly, effective) connectivity, ${ }^{4}$ a daring concept that Karl Friston created on his way to developing theoretical and computational tools for the analysis of functional images of the brain (Friston, 1994). Connectivity deals with ways for information to

\footnotetext{
${ }^{4}$ Karl Friston (1994) intended functional connectivity to expose temporal correlations between neurophysiological events-a descriptive approach without a priori specification that the originating structures would affect one another-whereas effective connectivity is meant to address explicit influences between brain parts (how much does A affect B, an estimation based on a priori model of those areas' relations, later reframed as causality).
}

go from one place to another. Ideally, we would be able to measure the connection (the "traffic" between two sites) independently from the state of those sites where said traffic imparts effect (as one would measure how many cars travel on the road between two cities). If independently measured, large scale connectivity and local activity would be amenable to reveal their effect on each other. Since we do not have adequate tools to measure the flow of information in living fiber tracts at large though, connectivity is not measured directly; rather it is inferred from the way brain components behave. Interaction, it is postulated, has to leave detectable traces in the behavior of its participants. Of course, contemporaneous theories have shaped the lens through which scientists have tried to see this influence. To make things practical, the assumption was often made that regions exchanging information must be correlated or synchronized (connectivity $\rightarrow$ correlation). Flipping things around for the operational goal of quantifying the unquantifiable information flow, "how much regions were correlated" became the proxy for how much they exchanged information (correlation $\rightarrow$ connectivity). But with only this concept of synchronization under the scope, we may see a mere fraction of the brain at work, the tip of the iceberg. What if most coordinated behaviors in the brain do not fall under our definition of correlation or synchrony? Depending on the methods used, that would mean for instance brain regions that are coordinated yet not temporally coincident; or assemblies in which self-organization favors a fluid coordination regime sans-attractor (such as metastable tendencies, to be discussed below) over rigid states of phase-locking. Can we see dynamics in which no absolute "order" emerges in space (synchronization) or in time (transfer), and still make sense of it as a means for the brain to function? Those are the dark and uncharted areas in the spatiotemporal organization of complex systems - those for which we sorely lack concepts and methods (Figure 2).

\section{ENLARGING THE SCOPE: METASTABILITY}

The set of questions above resonates with a recent shift in perspective on brain function, from a primary focus on neural synchronization to the broader-and deeper-problem of dynamic coordination. This shift was salient in the editorial introduction to a special issue of Nature Review Neuroscience in February 2010, where the word "coordination" occurred six times in a short text of 250 words (From The Editors, 2010). And this is a concept that is growing (Kelso, 1995, 2012; Bressler and Kelso, 2001; Kelso and Tognoli, 2007; Werner, 2007; Rabinovich et al., 2008; Tognoli and Kelso, 2009, 2013, 2014; von der Malsburg et al., 2010; Farmer, 2011; Kelso et al., 2013). Coordination includes synchronization as one possible collective behavior, but it also considers many other ways for components of the brain to interact. In particular, under certain conditions partially synchronized behaviors arise. In them, the parts exhibit simultaneous tendencies to temporarily couple and to segregate as independent entities. Such metastable regimes, we and others have shown, constitute a recipe for complexity (Kelso, 1995, 2012; Friston, 1997; Freeman and Holmes, 2005; Kelso and Tognoli, 2007; Werner, 2007; Rabinovich et al., 2008; Tognoli and Kelso, 2009, 2013, 2014; Bhowmik and Shanahan, 


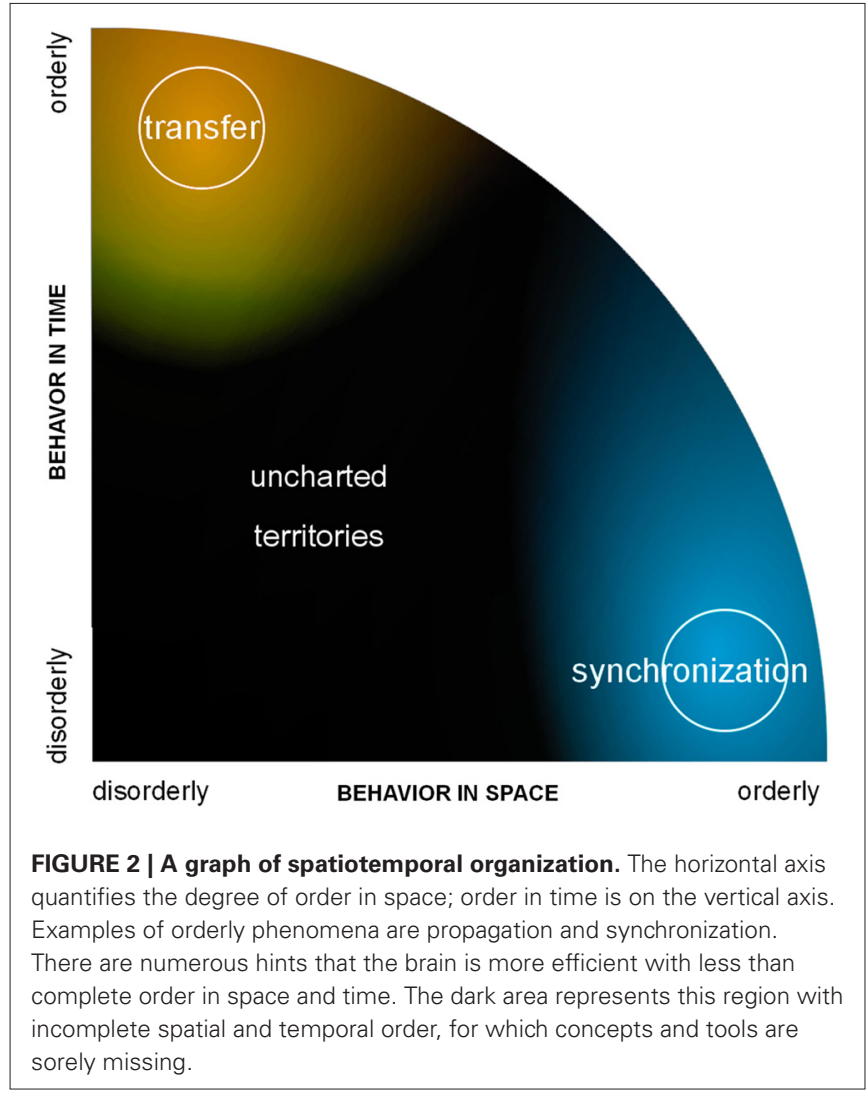

2013; Kelso et al., 2013). Why is metastable coordination dynamics of high interest to understanding how the brain works? First, it typically arises when the parts are no longer perfect clones of one another (e.g., as in computational models built from collections of identical neurons). When symmetry is broken and interacting parts are recognized in the diversity of their intrinsic behavior, a more realistic picture of brain function emerges (indeed a trend toward studying more diverse associations in the brain may explain the shift in perspective, for instance the interactions between neurons and astrocytes, see, e.g., Wade et al., 2013). Second, incomplete synchronization is more adaptive than pure forms. A fundamental nonlinearity in brain self-organization exists. Too much autonomy (parts of the brain hardly ever affected by what others are doing) prevents emergence, integration and mass action (Uhlhaas and Singer, 2006). Yet, too much integration (for instance the whole brain engaged in a giant common behavior, Cruikshank and Connors, 2008) is inadequate too, because the respective parts can no longer do what they are supposed to do in contributing to collective behavior. The parts then have no choice but to behave exactly like each other and the richness of their individual dispositions is lost to the ensemble. It is enough to note-as many have-that excess synchronization is pathological in the brain, for instance in epilepsy or Parkinson's disease (Uhlhaas and Singer, 2006; Hammond et al., 2007; Lehnertz et al., 2009). As a result, the ideal place for a brain to exhibit a rich set of meaningful behaviors is in-between integration and segregation. This is where the "incomplete" synchronization tendencies or metastable coordination comes into play (Tononi et al., 1994; Kelso, 1995, 2012; Friston, 1997; Werner, 2007; Chialvo, 2010; Tognoli and Kelso, 2013, 2014). Elsewhere, we have also speculated on the tremendous functional advantages that metastability would confer to a system, including speed, flexibility and resilience (Kelso and Tognoli, 2007; Kelso, 2012).

\section{CREATING A NEW CONCEPTUAL FRAMEWORK}

The gap between our current understanding of the brain and the miracles of our mental life and behavioral achievements (for example, consciousness and capacity for invention) remains abysmal. Looking through the history of science, several paradigms of brain function flourished and then dried up following the ebbs and flows of scientific metaphors. The ultimate model, the one that allows to forecast all matters of brain action and to design an artificial counterpart of multiple functional prowesses, remains out of sight. Two lines of thinking have been much explored in recent times: information transfer and synchronization. Their success owes much to the fact that they are special cases and open to quantification. When examined together though, they reveal some incompatibilities that seem to require a relaxation of both principles: less stringent temporal order and less complete spatial order. To advance our understanding of the brain, Neuroscience must open up avenues to study functional behavior in a broader sense. We face two alternatives: to leave it all within the current framework, with the approximate truth derived from current theories (the brain "sort of transfers information", and it operates with "near synchrony"), or to face the issue head on with a different theoretical mindset. In the latter case, a new phenomenology is up for grasp. It will be difficult to conceptualize, and even more so to observe, since it points toward a void in understanding. Modeling approaches can lead the way, by informing which observables we can expect to encounter in the coordinating living brain. And tools will have to be revised or built to adapt to this new world, tools that will say for instance, when "more synchrony" is "too much synchrony" (astonishingly, this simple question is not built into our current enquiries, despite obvious evidence of the ills of excess synchrony). We note that Brain Science is reaching a turning point that may make this renewal possible: it shows many signs of its readiness to enlarge the scope on brain function, not least of which is a recent outburst of interest in segregation phenomena (Poulet and Petersen, 2008; Ecker et al., 2010; Renart et al., 2010). A new paradigm would help to integrate principles that seem contradictory in their radical form: transfer and synchronization, as well as integration and segregation. Those pairs of concepts are reconciled under the dynamical regime of metastability (Kelso, 1995; Kelso and Engstrøm, 2006; Kelso and Tognoli, 2007; Tognoli and Kelso, 2014). Parts making-up the brain (for instance molecules, neurons, or brain areas) have simultaneous tendencies for independence and cooperation. As a result, they engage in the double-duty of lending their help to the collective behavior undertaken by several brain parts, and of performing their own independent behavior. Under a metastable regime, information is continuously created, 
preserved and annihilated by spatiotemporally changing coalitions among parts and processes. This is a source of dynamic complexity, and the likely origin of the human brain's many prowesses.

\section{ACKNOWLEDGMENTS}

This work was supported by NIMH (MH080838), NSF (BCS0826897), the US ONR (N00014-09-1-0527), the Davimos Family Endowment for Excellence in Science and the Chaire d'excellence Pierre de Fermat. Suggestions by both reviewers are gratefully acknowledged.

\section{REFERENCES}

(2010) From The Editors. Nature Reviews Neuroscience 11:71. doi: 10.1038/nrn2802 Ashby, W. R. (1962). "Principles of the self-organizing system," in Principles of SelfOrganization: Transactions of the University of Illinois Symposium, eds H. Von Foerster and G. W. Zopf, Jr. (London, UK: Pergamon Press), 255-278.

Barreto, E., So, P., Gluckman, B. J., and Schiff, S. J. (2000). From generalized synchrony to topological decoherence: emergent sets in coupled chaotic systems. Phys. Rev. Lett. 84, 1689-1692. doi: 10.1103/physrevlett.84.1689

Bhowmik, D., and Shanahan, M. (2013). Metastability and inter-band frequency modulation in networks of oscillating spiking neuron populations. PLoS One 8:e62234. doi: 10.1371/journal.pone.0062234

Bressler, S. L., and Kelso, J. A. S. (2001). Cortical coordination dynamics and cognition. Trends Cogn. Sci. 5, 26-36. doi: 10.1016/s1364-6613(00)01564-3

Bressler, S. L., Richter, C. G., Chen, Y., and Ding, M. (2007). Cortical functional network organization from autoregressive modeling of local field potential oscillations. Stat. Med. 26, 3875-3885. doi: 10.1002/sim.2935

Bressler, S. L., and Seth, A. K. (2011). Wiener-granger causality: a well established methodology. Neuroimage 58, 323-329. doi: 10.1016/i.neuroimage.2010.02.059

Bressler, S. L., and Tognoli, E. (2006). Operational principles of neurocognitive networks. Int. J. Psychophysiol. 60, 139-148. doi: 10.1016/j.ijpsycho.2005.12.008

Buzsáki, G. (2007). The structure of consciousness. Nature 446:267. doi: 10. $1038 / 446267 \mathrm{a}$

Catani, M., and ffytche, D. H. (2005). The rises and falls of disconnection syndromes. Brain 128, 2224-2239. doi: 10.1093/brain/awh622

Charcot, J. M. (1878). Leçons sur les Localisations dans les Maladies du Cerveau. Paris: Delahaye et Co.

Chialvo, D. R. (2010). Emergent complex neural dynamics. Nat. Phys. 6, 744-750. doi: $10.1038 /$ nphys 1803

Cruikshank, S. J., and Connors, B. W. (2008). State-sanctioned synchrony. Nature 454, 839-840. doi: 10.1038/454839a

Eccles, J. C. (1966). The ionic mechanisms of excitatory and inhibitory synaptic action. Ann. N Y Acad. Sci. 137, 473-494. doi: 10.1111/j.1749-6632.1966. tb50176.x

Ecker, A. S., Berens, P., Keliris, G. A., Bethge, M., Logothetis, N. K., and Tolias, A. S. (2010). Decorrelated neuronal firing in cortical microcircuits. Science 327, 584-587. doi: 10.1126/science. 1179867

Eckhorn, R., Bauer, R., Jordan, W., Brosch, M., Kruse, W., Munk, M., et al. (1988). Coherent oscillations: a mechanism of feature linking in the visual cortex? Multiple electrode and correlation analyses in the cat. Biol. Cybern. 60, 121-130. doi: 10.1007/bf00202899

Edelman, G. M. (1993). Neural Darwinism: selection and reentrant signaling in higher brain function. Neuron 10, 115-125. doi: 10.1016/0896-6273(93)90304-a

Eigen, M., and Schuster, P. (1979). The Hypercycle: A Principle of Natural Selforganisation. Heidelberg: Springer-Verlag.

Farmer, S. (2011). Review of dynamic coordination in the brain: from neurons to mind. J. Psychophysiol. 25, 201-202. doi: 10.1027/0269-8803/a000055

Freeman, W. J. (1975). Mass Action in the Nervous System. New York: Academic Press.

Freeman, W. J., and Holmes, M. D. (2005). Metastability, instability, and state transition in neocortex. Neural Netw. 18, 497-504. doi: 10.1016/j.neunet.2005. 06.014

Fries, P. (2005). A mechanism for cognitive dynamics: neuronal communication through neuronal coherence. Trends Cogn. Sci. 9, 474-480. doi: 10.1016/j.tics. 2005.08.011
Friston, K. J. (1994). Functional and effective connectivity in neuroimaging: a synthesis. Hum. Brain Mapp. 2, 56-78. doi: 10.1002/hbm.460020107

Friston, K. J. (1997). Transients, metastability and neuronal dynamics. Neuroimage 5, 164-171. doi: 10.1006/nimg.1997.0259

Gallos, L. K., Sigman, M., and Makse, H. A. (2012). The conundrum of functional brain networks: small-world efficiency or fractal modularity. Front. Physiol. 3:123. doi: 10.3389/fphys.2012.00123

Gray, C. M., and Singer, W. (1989). Stimulus-specific neuronal oscillations in orientation columns of cat visual cortex. Proc. Natl. Acad. Sci. U S A 86, 1698 1702. doi: $10.1073 /$ pnas.86.5.1698

Grossberg, S. (1976). Adaptive pattern classification and universal recoding: I. Parallel development and coding of neural feature detectors. Biol. Cybern. 23, 121-134. doi: 10.1007/bf00344744

Haken, H. (1983). Synergetics, an Introduction: Nonequilibrium Phase Transitions and Self-Organization in Physics, Chemistry and Biology. New York: SpringerVerlag.

Hammond, C., Bergman, H., and Brown, P. (2007). Pathological synchronization in Parkinson's disease: networks, models and treatments. Trends Neurosci. 30, 357-364. doi: 10.1016/j.tins.2007.05.004

Hebb, D. O. (1949). The Organization of Behavior. New-York: John Wiley.

Hodgkin, A. L., and Huxley, A. F. (1952). A quantitative description of membrane current and its application to conduction and excitation in nerve. J. Physiol. 117, 500-544.

Honey, C. J., Kötter, R., Breakspear, M., and Sporns, O. (2007). Network structure of cerebral cortex shapes functional connectivity on multiple time scales. Proc. Natl. Acad. Sci. U S A 104, 10240-10245. doi: 10.1073/pnas.07015 19104

Honey, C. J., Thivierge, J. P., and Sporns, O. (2010). Can structure predict function in the human brain? Neuroimage 52, 766-776. doi: 10.1016/j.neuroimage. 2010 01.071

Hopfield, J. J. (1982). Neural networks and physical systems with emergent collective computational abilities. Proc. Natl. Acad. Sci. U S A 79, 2554-2558. doi: 10. 1073/pnas.79.8.2554

Kelso, J. A. S. (1995). Dynamic Patterns: The Self-Organization of Brain and Behavior. Cambridge, Massachusetts: The MIT Press.

Kelso, J. A. S. (2008). An essay on understanding the mind: the A.S. Iberall Lecture. Ecol. Psychol. 20, 180-208. doi: 10.1080/10407410801949297

Kelso, J. A. S. (2012). Multistability and metastability: understanding dynamic coordination in the brain. Philos. Trans. R. Soc. Lond. B Biol. Sci. 367, 906-918. doi: 10.1098/rstb.2011.0351

Kelso, J. A. S., and Engstrøm, D. A. (2006). The Complementary Nature. Cambridge, MA: The MIT Press.

Kelso, J. A. S., Dumas, G., and Tognoli, E. (2013). Outline of a general theory of behavior and brain coordination. Neural Netw. 37, 120-131. doi: 10.1016/j. neunet.2012.09.003

Kelso, J. A. S., and Haken, H. (1995). "New laws to be expected in the organism: synergetics of brain and behavior," in What is Life? The Next Fifty Years: Speculations on the Future of Biology, eds M. P. Murphy and L. A. J. O’Neill (Cambridge, UK: Cambridge University Press), 137-160.

Kelso, J. A. S., and Tognoli, E. (2007). "Toward a complementary neuroscience: metastable coordination dynamics of the brain," in Neurodynamics of Higherlevel Cognition and Consciousness, eds R. Kozma and L. Perlovsky (Heidelberg: Springer), 39-59.

Kelso, J. A. S., and Tuller, B. (1981). Toward a theory of apractic syndromes. Brain Lang. 12, 224-245. doi: 10.1016/0093-934x(81)90016-x

Kostrubiec, V., Zanone, P.-G., Fuchs, A., and Kelso, J. A. S. (2012). Beyond the blank slate: routes to learning new coordination patterns depend on the intrinsic dynamics of the learner - experimental evidence and theoretical model. Front. Hum. Neurosci. 6:222. doi: 10.3389/fnhum.2012.00222

Lehnertz, K., Bialonski, S., Horstmann, M. T., Krug, D., Rothkegel, A., Staniek, M., et al. (2009). Synchronization phenomena in human epileptic brain networks. J. Neurosci. Methods 183, 42-48. doi: 10.1016/j.jneumeth.2009. 05.015

McLeod, P., Plunkett, K., and Rolls, E. T. (1998). Introduction to Connectionist Modelling of Cognitive Processes. Oxford, UK, New-York, NY: Oxford University Press.

Meunier, D., Lambiotte, R., and Bullmore, E. T. (2010). Modular and hierarchically modular organization of brain networks. Front. Neurosci. 4:200. doi: 10 . 3389/fnins.2010.00200 
Poulet, J. F. A., and Petersen, C. C. H. (2008). Internal brain state regulates membrane potential synchrony in barrel cortex of behaving mice. Nature 454, 881-885. doi: 10.1038/nature07150

Rabinovich, M. I., Huerta, R., Varona, P., and Afraimovich, V. S. (2008). Transient cognitive dynamics, metastability and decision making. PLoS Comput. Biol. 4:e1000072. doi: 10.1371/journal.pcbi.1000072

Renart, A., de la Rocha, J., Bartho, P., Hollender, L., Parga, N., Reyes, A., et al. (2010). The asynchronous state in cortical circuits. Science 327, 587-590. doi: 10. $1126 /$ science. 1179850

Robert, A. (1999). Lamination and Within-Area Integration in the Neocortex. San Diego: University of California, Doctoral dissertation.

Rulkov, N. F., Sushchik, M. M., Tsimring, L. S., and Abarbanel, H. D. (1995). Generalized synchronization of chaos in directionally coupled chaotic systems. Phys. Rev. E Stat. Phys. Plasmas Fluids Relat. Interdiscip. Topics 51, 980-994. doi: $10.1103 /$ physreve.51.980

Rumelhart, D. E., McClelland, J. L., and The PDP Research Group (1986). Parallel Distributed Processing: Explorations in the Microstructure of Cognition, Volume 1: Foundations. Cambridge, MA: MIT Press.

Shannon, C. E. (1948). A mathematical theory of communication. Bell Syst. Tech. J. 27, 623-656. doi: 10.1002/j.1538-7305.1948.tb00917.x

Sperry, R. W. (1969). A modified concept of consciousness. Psychol. Rev. 76, 532 536. doi: 10.1037/h0028156

Sporns, O., Chialvo, D. R., Kaiser, M., and Hilgetag, C. C. (2004). Organization, development and function of complex brain networks. Trends Cogn. Sci. 8, 418425. doi: 10.1016/j.tics.2004.07.008

Sporns, O., Tononi, G., and Edelman, G. M. (2000). Theoretical neuroanatomy: relating anatomical and functional connectivity in graphs and cortical connection matrices. Cereb. Cortex 10, 127-141. doi: 10.1093/cercor/10.2.127

Tognoli, E., and Kelso, J. A. S. (2009). Brain coordination dynamics: true and false faces of phase synchrony and metastability. Prog. Neurobiol. 87, 31-40. doi: 10. 1016/j.pneurobio.2008.09.014

Tognoli, E., and Kelso, J. A. S. (2013). "On the brain's dynamical complexity: coupling and causal influences across spatiotemporal scales," in Advances in Cognitive Neurodynamics (III), ed Y. Yamaguchi (Netherlands: Springer), 259-265.

Tognoli, E., and Kelso, J. A. S. (2014). The metastable brain. Neuron 81, 35-48. doi: 10.1016/j.neuron.2013.12.022

Tononi, G., Sporns, O., and Edelman, G. M. (1994). A measure for brain complexity: relating functional segregation and integration in the nervous system. Proc. Natl. Acad. Sci. U S A 91, 5033-5037. doi: 10.1073/pnas.91. 11.5033

Uhlhaas, P. J., and Singer, W. (2006). Neural synchrony in brain disorders: relevance for cognitive dysfunctions and pathophysiology. Neuron 52, 155-168. doi: 10 . 1016/j.neuron.2006.09.020

Varela, F., Lachaux, J.-P., Rodriguez, E., and Martinerie, J. (2001). The brainweb: phase synchronization and large-scale integration. Nat. Rev. Neurosci. 2, 229239. doi: $10.1038 / 35067550$

von der Malsburg, C., Phillips, W. A., and Singer, W. (Eds). (2010). Dynamic Coordination in the Brain: From Neurons to Mind, Strüngmann Forum Report, Vol. 5. Cambridge, MA: MIT Press.

Wade, J. J., McDaid, L. J., Harkin, J., Crunelli, V., and Kelso, J. A. S. (2013). Biophysically-based computational models of astrocyte $\sim$ neuron coupling and their functional significance. Front. Comput. Neurosci. 7:44. doi: 10.3389/fncom. 2013.00044

Werner, G. (2007). Metastability, criticality and phase transitions in brain and its models. Biosystems 90, 496-508. doi: 10.1016/j.biosystems.2006.12.001

Yates, E. (2008). Homeokinetics/Homeodynamics: a physical heuristic for life and complexity. Ecol. Psychol. 20, 148-179. doi: 10.1080/10407410801977546

Zilles, K., Palomero-Gallagher, N., Grefkes, C., Scheperjans, F., Boy, C., Amunts, K., et al. (2002). Architectonics of the human cerebral cortex and transmitter receptor fingerprints: reconciling functional neuroanatomy and neurochemistry. Eur. Neuropsychopharmacol. 12, 587-599. doi: 10.1016/s0924-977x(02)00108-6

Conflict of Interest Statement: The authors declare that the research was conducted in the absence of any commercial or financial relationships that could be construed as a potential conflict of interest.

Received: 20 March 2014; accepted: 09 June 2014; published online: 25 June 2014. Citation: Tognoli E and Kelso JAS (2014) Enlarging the scope: grasping brain complexity. Front. Syst. Neurosci. 8:122. doi: 10.3389/fnsys.2014.00122

This article was submitted to the journal Frontiers in Systems Neuroscience.

Copyright (c) 2014 Tognoli and Kelso. This is an open-access article distributed under the terms of the Creative Commons Attribution License (CC BY). The use, distribution or reproduction in other forums is permitted, provided the original author(s) or licensor are credited and that the original publication in this journal is cited, in accordance with accepted academic practice. No use, distribution or reproduction is permitted which does not comply with these terms. 